\title{
Ferroelectric phase transition of stoichiometric lithium tantalate studied by Raman, Brillouin, and neutron scattering
}

\author{
Anwar Hushur, ${ }^{1}$ Severian Gvasaliya, ${ }^{2, *}$ Bertrand Roessli, ${ }^{2}$ Sergey Lushnikov, ${ }^{3}$ and Seiji Kojima ${ }^{1, \dagger}$ \\ ${ }^{1}$ Graduate School of Pure and Applied Sciences, University of Tsukuba, Tsukuba, Ibaraki 305-8573, Japan \\ ${ }^{2}$ Laboratory for Neutron Scattering, ETHZ and Paul-Scherrer Institut, CH-5232 Villigen PSI, Switzerland \\ ${ }^{3}$ Ioffe Physical Technical Institute, 26 Politekhnicheskaya, 194021 St. Petersburg, Russia
}

(Received 17 June 2006; published 7 August 2007)

\begin{abstract}
The ferroelectric phase transition of nearly stoichiometric $\mathrm{LiTaO}_{3}$ crystals with very low lithium deficiency has been studied by Raman, Brillouin, and neutron scattering. The neutron elastic scattering shows the high value of the Curie temperature $T_{C}=960 \mathrm{~K}$ in comparison with $T_{C}=873 \mathrm{~K}$ of the congruent crystals. In order to make clear the order-disorder character near the Curie temperature, the central peak (CP) (quasielastic scattering) has been investigated by the broadband Brillouin scattering and low-frequency Raman scattering in the wide temperature range of 300-1067 K. In Brillouin and Raman scattering, CP is observed at the polarized scattering geometry in all the temperatures studied. The polarized nature of $\mathrm{CP}$ indicates that its origin is the polarization fluctuation along the ferroelectric $c$ axis. It is found that the relaxation time determined from the width of CP clearly shows a critical slowing down of an order-disorder phase transition in the vicinity of $T_{C}$.
\end{abstract}

DOI: 10.1103/PhysRevB.76.064104

PACS number(s): 63.70.+h, 77.80.Bh, 77.84.Dy, 78.30.-j

\section{INTRODUCTION}

The dynamics of structural phase transitions in ferroelectric materials has been a hot topic for the solid-state physics. However, there are still unsolved problems on the nature of ferroelectric phase transitions, displacive or order-disorder type. The one side of this problem comes from the condensation of a low-frequency optic soft-mode picture of structural phase transitions based on the Cochran's model. ${ }^{1}$ According to the mean-field approximation, a second-order displacive phase transition is associated with a soft phonon, and its frequency $\omega_{s}$ goes to zero as the temperature approaches a transition temperature $T_{C}{ }^{1,2}$

$$
\omega^{2} \propto T_{C}-T \quad\left(T<T_{C}\right) .
$$

In contrast, inelastic scattering spectra on various ferroelectric materials show a central peak (CP) in addition to the soft modes. ${ }^{2-4}$ The first experimental evidence ${ }^{3}$ for the deviation from the soft-mode picture of a structural phase transition was reported in neutron scattering experiments of $\mathrm{SrTiO}_{3}$ and later in Raman scattering experiments of $\mathrm{KH}_{2} \mathrm{PO}_{4} \cdot{ }^{4} \mathrm{CP}$ reflects some kinds of microscopic relaxation process related to the ferroelectric polarization. In this case, the intensity of phonon modes as well as their mode frequency remains at a finite value as $T \rightarrow T_{C}$, while the intensity and linewidth of a relaxation mode diverges near $T_{C}$. The temperature dependence of relaxation time $\tau$ of a relaxation mode shows a critical slowing down with a characteristic frequency $\tau_{0}{ }^{2}$

$$
\tau=\frac{\tau_{0} T_{C}}{\left|T-T_{C}\right|} .
$$

NMR study ${ }^{5}$ shows the coexistent of order-disorder and displacive components in the phase transition mechanism of $\mathrm{BaTiO}_{3}$, which had been considered as a typical example of the soft-mode concept. There is one particular class of ferroelectric crystals, the lithium niobate $\mathrm{LiNbO}_{3}$ (LN) and lithium tantalate $\mathrm{LiTaO}_{3}(\mathrm{LT})$; in spite of the numerous studies in the last 40 years, the phase transition mechanism of these materials is still controversial, because most studies used crystals with the congruent composition and the deficiency of lithium ions of about $3 \mathrm{~mol} \%$ obscures the dynamical properties near $T_{C}$.

LN and LT crystals are piezoelectric and ferroelectric at room temperature (RT) and have rich applications in electromechanical devices, i.e., surface acoustic filters, ultrasonic transducers, and optical devices such as electro-optic, nonlinear optic, and photorefractive devices. ${ }^{6-8}$ The lowfrequency optic phonons of LN and LT were studied extensively because of their importance in nonlinear optical, electro-optical, and piezoelectric properties. It is well known that LT of the congruent composition undergoes a ferroelectric (second-order) phase transition at about $T_{C}=873 \mathrm{~K}$ from a high temperature paraelectric (PE) phase with the point group $\overline{3} m\left(D_{3 d}\right)$ to the low temperature ferroelectric (FE) phase with the point group $3 m\left(C_{3 v}\right)$. It is uniaxial at all temperatures; therefore, the ferroelectric soft mode is nondegenerate both above and below the Curie temperature $T_{C}$. The displacement can be represented by a single order parameter, and it describes the basic nature of the ferroelectric phase transition of LT. Thus, it is expected that LT can be one of the examples of the simplest ferroelectric transition and was chosen as a standard sample to apply the statistical theory.

In a ferroelectric phase, the direction of a spontaneous polarization is along the threefold $c$ axis, and the soft optic mode with $A_{1}(z)$ symmetry has been reported. Although it becomes overdamped far below $T_{C}$, they reported that the phonon frequency of $A_{1}(z)$ mode shows softening on approaching $T_{C}$ from below according to Eq. (1). ${ }^{9}$ According to the theoretical analysis of many experimental data, Lines concluded that the phase transition of $\mathrm{LiTaO}_{3}$ is the intermediate between displacive and order-disorder. ${ }^{10}$ Penna et al. found a Debye-like CP in a FE phase and anomalous polariton dispersion of $A_{1}(z)$ phonon modes near $T_{C}$ and suggested an order-disorder nature of this ferroelectric phase transition. ${ }^{11,12}$ Servoin and Gervais also reported the soft 
phonon in both phases by the infrared reflectivity measurement. ${ }^{13}$ It was suggested that the $\mathrm{CP}$ in Raman scattering is not a central mode which occurs in addition to onephonon spectral features. It is rather one of the $4 A_{1}$ normal modes, which becomes nearly soft and heavily overdamped near $T_{C}$. The low-frequency Raman study showed not only a soft phonon mode but also CP related to the order-disorder nature, and the increase of relaxation time up to 2.5 ps below $T_{C}$ was reported. ${ }^{14}$ On the other hand, Raptis studied the lattice modes of LT in both phases by Raman scattering, while no evidence of softening was found. ${ }^{15}$ Recent hyperRaman, Raman, and inelastic neutron scattering studies of LT preferred order-disorder than the displacive model. ${ }^{16,17}$ The neutron scattering study shows that the Ta ion might move to the center of its oxygen cage on approaching $T_{C}$, while the $\mathrm{Li}$ ion did not enter its centrosymmetric oxygen plane but took positions $+0.037 \mathrm{~nm}$ on either side of this plane with equal probability. ${ }^{18}$ This fact indicates that the double well potential of $\mathrm{Li}$ ions probably plays a dominant role of an order-disorder nature. The existence of a relaxation mode at room temperature was also reported by the impulsive stimulated Raman scattering, the terahertz time domain spectroscopy, and low-frequency dielectric measurements. ${ }^{19-22}$

Up to the present, in several investigations, the orderdisorder nature was reported; nevertheless, there is no clear observation of the critical slowing down near the vicinity of $T_{C}$ given by Eq. (2). One possible reason is that all the investigations used congruent $\mathrm{LiTaO}_{3}$ crystals (CLT) which include the high defect density at Li site due to the deficiency of Li. Considering high defect density at Li site of about $3 \mathrm{~mol} \%$ in CLT, there is the possibility that the orderdisorder nature is smeared by such defects. Recently, nearly stoichiometric lithium tantalate (SLT) crystals are available by the crystal growth using the double crucible Czochralski (DCCZ) method, and their $T_{C}$ is about $90 \mathrm{~K}$ higher than that of CLT. Then, in SLT, we can expect the clear observation of critical slowing down related to the order-disorder nature of ferroelectricity. Another reason is that the low-frequency limit of the previous studies is rather high; therefore, misestimating of CP width and the defect mode observed at around $60 \mathrm{~cm}^{-1}$ near $T_{C}$ cause significant errors in the study of CP. Thus, we studied SLT crystals in the lowest-frequency range down to $0.1 \mathrm{~cm}^{-1}$ by Brillouin, Raman, and neutron scattering.

\section{EXPERIMENT}

Nearly stoichiometric $\mathrm{LiTaO}_{3}$ (SLT) crystals of high optical quality grown by DCCZ method were purchased from Oxide Corporation. The nominal $\left[\mathrm{Li}_{2} \mathrm{O}\right]:\left[\mathrm{Ta}_{2} \mathrm{O}_{5}\right]$ mole ratio is $49.9: 50.1(\mathrm{~mol} \%)$. Samples are parallelepiped $1.5 \times 4$ $\times 5$ and $3 \times 4 \times 5 \mathrm{~mm}^{3}$ along crystallographic $a, b$, and $c$ axes for light scattering, and $9 \times 10 \times 11 \mathrm{~mm}^{3}$ for neutron scattering. The six surfaces are polished to optical grade.

Brillouin scattering was measured by a $3+3$ pass tandem Fabry-Pérot interferometer of high contrast and high resolution and a compact cylinder furnace especially designed for light scattering. Brillouin scattering was excited by an $\mathrm{Ar}^{+}$

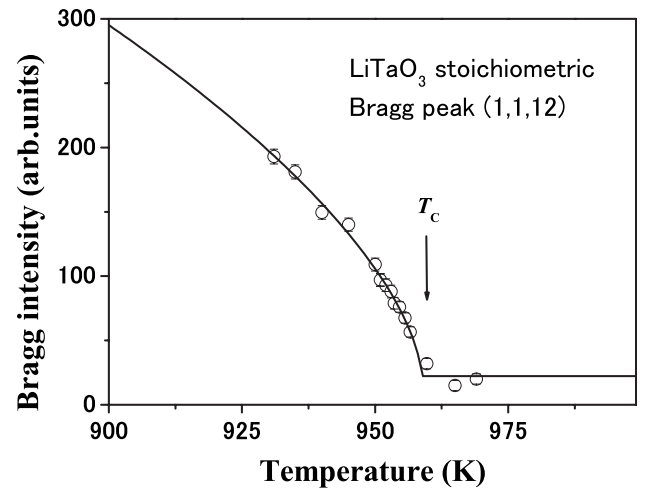

FIG. 1. The integrated intensity of the $(1,1,12)$ Bragg peak of the neutron diffraction experiments in a stoichiometric $\mathrm{LiTaO}_{3}$ single crystal as a function of temperature. The solid line indicates the curve fitted by the critical form $A\left(T_{C}-T\right)^{2 \beta}$, with parameters $T_{C}=960.2 \pm 0.4 \mathrm{~K}$ and $2 \beta=0.71 \pm 0.01$.

ion laser with a wavelength of $514.5 \mathrm{~nm}$ and power of $100 \mathrm{~mW}$. A backscattering geometry of the experiments was employed. A conventional photon-counting system and multichannel analyzer were used to acquire and to average output signals. The sample was put in the furnace with temperature variations up to $1373 \mathrm{~K}$ and stability of $\pm 0.5 \mathrm{~K}$. The $a(c, c) \bar{a}$ and $a(c, b) \bar{a}$ geometries were used for polarized and depolarized scattering, respectively. The Brillouin spectra were measured with FSR of $200 \mathrm{GHz}$ and the scan range of $450 \mathrm{GHz}^{23}$

Raman scattering is excited using a diode-pumped solidstate laser with a wavelength of $532 \mathrm{~nm}$ and power of about $40 \mathrm{~mW}$. Scattered light from a sample is collected at the right angle scattering geometry and analyzed by a triplegrating spectrometer of additive dispersion (Jobin Yvon T64000). The spectral resolution was about $2 \mathrm{~cm}^{-1}$. All the spectra were measured in the range of $-350-1000 \mathrm{~cm}^{-1}$ at two different scattering geometries, $a(c, c) b$ and $a(c, a) b$, in the wide temperature range from RT to $1023 \mathrm{~K}$. The Rayleigh line contribution from the laser is very small and restricted in the range from -4 to $4 \mathrm{~cm}^{-1}$. The high temperature furnace used for Brillouin scattering is the same as that for Raman scattering.

Using the elastic and inelastic neutron scattering measurements, we investigated the static and dynamical properties of nearly stoichiometric $\mathrm{LiTaO}_{3}$ in the temperature range from 300 to $1100 \mathrm{~K}$. The experiments were performed at the coldneutron three-axis spectrometer TASP, ${ }^{24}$ located at the neutron spallation source SINQ (Ref. 25) at the Paul-Scherrer Institut, Switzerland.

\section{RESULTS AND DISCUSSION}

\section{A. Neutron diffraction}

The ferroelectric phase transition was studied by neutron diffraction experiments. Figure 1 shows the temperature dependence of the $(1,1,12)$ Bragg peak intensity. Usually, we assume that the integrated intensity of the Bragg peak is proportional to the square of the order parameter and decreases gradually as the temperature approaches $T_{C}$ from be- 


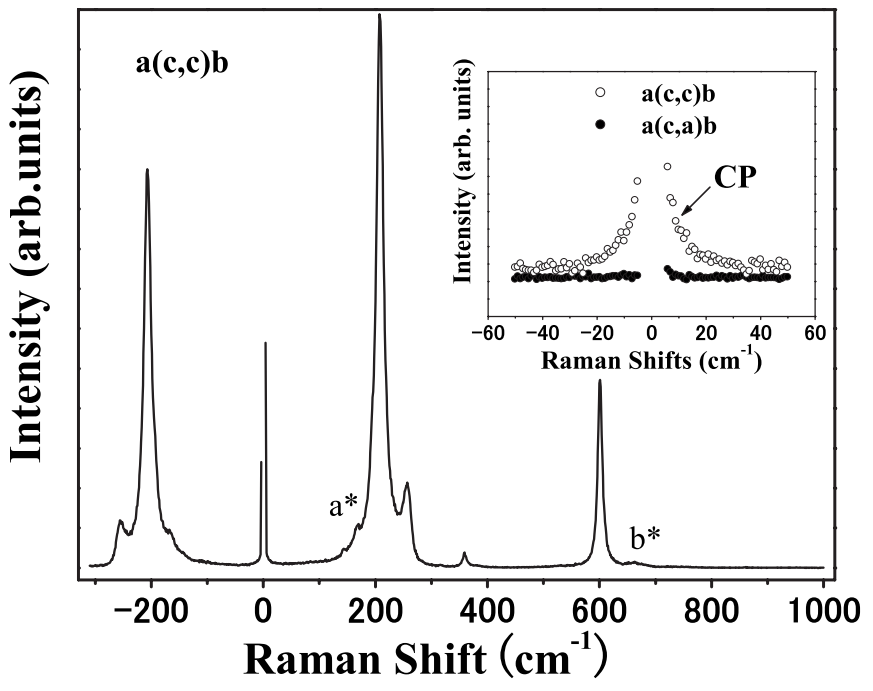

FIG. 2. The Raman spectrum of $a(c, c) b$ geometry recorded at room temperature in a stoichiometric $\mathrm{LiTaO}_{3}$ single crystal. It shows four $A_{1}(\mathrm{TO})$ first-order phonon peaks, an unspecified phonon band $\left(a^{*}\right)$, and a second-order phonon band $\left(b^{*}\right)$. Inset: the lowfrequency $\left(-50 \sim 50 \mathrm{~cm}^{-1}\right)$ spectra at room temperature at the $a(c, c) b$ and $a(c, a) b$ scattering geometries. CP means the central peak.

low. A second-order phase transition displays such a behavior in conventional models for cooperative behavior. We fit the Bragg intensity data to the critical form $A\left(T_{C}-T\right)^{2 \beta}$. The solid line in Fig. 1 is obtained from fitting with parameters $T_{C}=960.2 \pm 0.4 \mathrm{~K}$ and $2 \beta=0.71 \pm 0.01$. It is known that $T_{C}$ of LT depends on the stoichiometry of a crystal. The high $T_{C}$ $=960.2 \mathrm{~K}$ indicates nearly stoichiometric character of LT in our investigation.

\section{B. Optical phonon modes}

The symmetry of the optical modes of LT at the $\Gamma$ point of a reciprocal lattice space is given by $4 A_{1}+9 E+5 A_{2}$, where $A_{1}$ and $E$ modes are Raman- and infrared-active polar phonons while $A_{2}$ modes are Raman-active and infraredinactive nonpolar phonons. The spectra of $A_{1}(z)$ phonon modes were measured for SLT at RT from -300 to $1000 \mathrm{~cm}^{-1}$ at the $a(c, c) b$ scattering geometry as shown in Fig. 2. The inset of Fig. 2 shows the polarized and depolarized low-frequency spectra of $\mathrm{CP}$ from -50 to $50 \mathrm{~cm}^{-1}$ measured at $a(c, c) b$ and $a(c, a) b$ scattering geometries, respectively. The polarized CP was clearly observed at RT. As expected from the group theory, four peaks are detected at 208, 256, 359, and $601 \mathrm{~cm}^{-1}$. They are labeled as $A_{1}\left(\mathrm{TO}_{1}\right)$, $A_{1}\left(\mathrm{TO}_{2}\right), A_{1}\left(\mathrm{TO}_{3}\right)$, and $A_{1}\left(\mathrm{TO}_{4}\right)$, respectively. Shoulders of $A_{1}\left(\mathrm{TO}_{1}\right)$ and $A_{1}\left(\mathrm{TO}_{4}\right)$ are also observed in the spectrum. The scattering around $663 \mathrm{~cm}^{-1}$ is probably caused by twophonon scattering processes as discussed in the previous studies of CLT. ${ }^{15,24}$ The origin of the scattering around $167 \mathrm{~cm}^{-1}$ is, up to now, unknown. The normal modes observed in our spectrum at RT are similar to those of CLT previously reported, except for the phonon frequency and damping. It is known that several properties of LT depend on the lithium composition. The phonon frequency exhibits only a small dependence on the lithium composition of a crystal, while the phonon damping is very sensitive to it, the same as the case of LN. ${ }^{26,29}$ In the present study, the damping of $A_{1}\left(\mathrm{TO}_{1}\right)$ mode is $15 \mathrm{~cm}^{-1}$ for SLT, while for CLT it is $25 \mathrm{~cm}^{-1}$. The Raman spectra at RT are noteworthy from the two aspects in comparison with those previously reported. $9,15,16,27$ First, the defect mode of CLT observed around $90 \mathrm{~cm}^{-1}$ in previous study is absent in our spectra. This is attributed to the nearly stoichiometric composition of our sample. This defect mode frequency of CLT comes close to around $60 \mathrm{~cm}^{-1}$ on heating and gives misestimation to the relaxation time at high temperature (below $\left.T_{C}\right) .{ }^{16}$ Second, the polarized $\mathrm{CP}$ is clearly shown at RT. ${ }^{28}$

\section{Central peak}

CP observed at RT gives a relaxation time of approximately $0.8 \mathrm{ps}$ for SLT. The appearance of the fast relaxation process at RT was also reported as the anomalous increase in polariton damping rate in single- and multiple-pulse stimulated Raman scattering measurements. ${ }^{19,20}$ Araujo et al. observed the low-frequency dielectric dispersion for CLT at RT, and far below the $T_{C} \cdot{ }^{22}$ The experimental data at various temperatures can be well fitted by a Debye relaxation function. Kojima et al. determined the complex dielectric constants $\varepsilon_{z}(\omega)$ and $\varepsilon_{x}(\omega)$ of CLT in the sub terahertz to terahertz regions. ${ }^{21}$ Both the real and imaginary parts of $\varepsilon_{x}(\omega)$ are well reproduced only by a damped harmonic oscillator model. However, those of $\varepsilon_{z}(\omega)$ indicate the excess part, which may be attributed to the relaxation mode related to $\mathrm{CP}$ observed in our spectra.

The temperature dependence of the $A_{1}$ spectra was measured as shown in Fig. 3. Several features can be directly deduced from the temperature dependence. All the $A_{1}$ modes and the lowest $a^{*}$ mode show softening on heating. The $A_{1}\left(\mathrm{TO}_{1}\right)$ mode intensity at RT is very strong in comparison with those of $a^{*}$ and $A_{1}\left(\mathrm{TO}_{2}\right)$ modes. Upon heating, the intensity of these two modes and CP increases, whereas the $A_{1}\left(\mathrm{TO}_{1}\right)$ mode intensity decreases. Above $823 \mathrm{~K}$, the $a^{*}$ and $A_{1}\left(\mathrm{TO}_{1}\right)$ modes become very weak, and only the $A_{1}\left(\mathrm{TO}_{2}\right)$ mode is visible in a spectrum below $300 \mathrm{~cm}^{-1}$. In the spectrum from -300 to $300 \mathrm{~cm}^{-1}$ at $873 \mathrm{~K}$, only the strong $\mathrm{CP}$ and the $A_{1}\left(\mathrm{TO}_{2}\right)$ mode are observed. Therefore, it is difficult to correctly evaluate the temperature dependence of the $a^{*}$ and $A_{1}\left(\mathrm{TO}_{1}\right)$ modes near $T_{C}$. In this paper, we concentrate on the properties of CP near $T_{C}$. The behaviors of the acoustic and optic phonons will be published separately. Figures 4 and 5 show the Brillouin and Raman spectra in the vicinity of $T_{C}$. Upon heating, it is found that the intensity of $\mathrm{CP}$ and its linewidth increase markedly as the temperature approaches $T_{C}$.

\section{Analysis of central peaks}

In the damped oscillator model, the spectral response of optical modes $S(\nu, T)$ is given by 

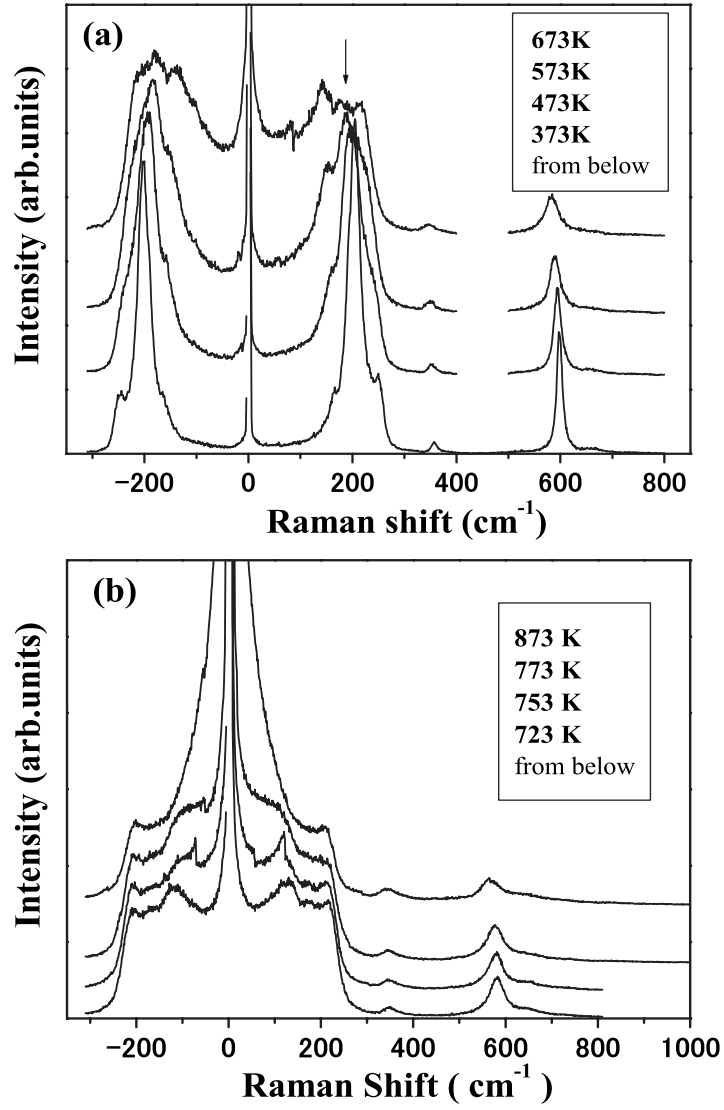

FIG. 3. The temperature dependence of the spectra measured at the $a(c, c) b$ scattering geometry. The arrows mark the soft phonon at $673 \mathrm{~K}$. The soft phonon mode and $a^{*}$ mode of Fig. 2 are invisible in the spectrum at $873 \mathrm{~K}$.

$$
S(\nu, T)=\chi^{\prime \prime}(\nu) F(\nu, T)=\sum_{i} \frac{f_{i} \nu \Gamma_{i}}{\left(\nu_{i}^{2}-\nu^{2}\right)^{2}+\nu^{2} \Gamma_{i}^{2}} F(\nu, T),
$$

where $\chi^{\prime \prime}(\nu)$ is the imaginary part of the dynamical susceptibility, and $\nu_{i}, \Gamma_{i}$, and $f_{i}$ are the phonon frequency, the

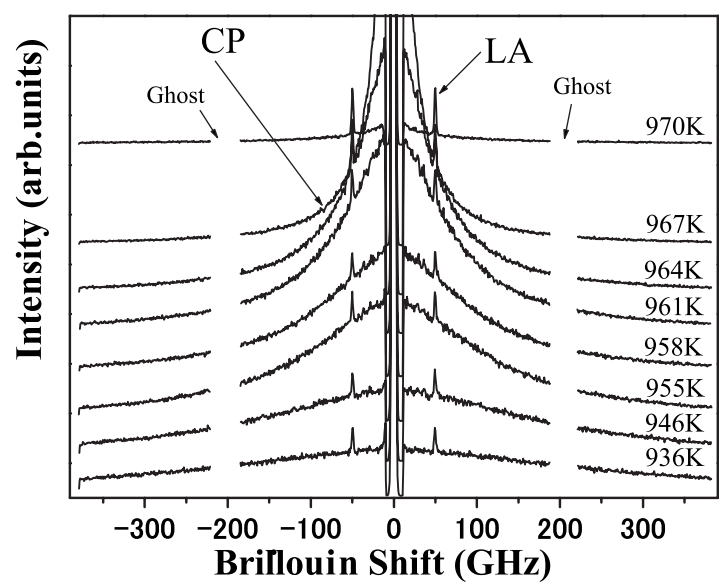

FIG. 4. Temperature dependence of the Brillouin spectra at the $a(c, c) \bar{a}$ scattering geometry. CP means central peak; LA means longitudinal acoustic phonon. We note that $\mathrm{CP}$ increases markedly toward $T_{C}$ and still exists above $T_{C}$.

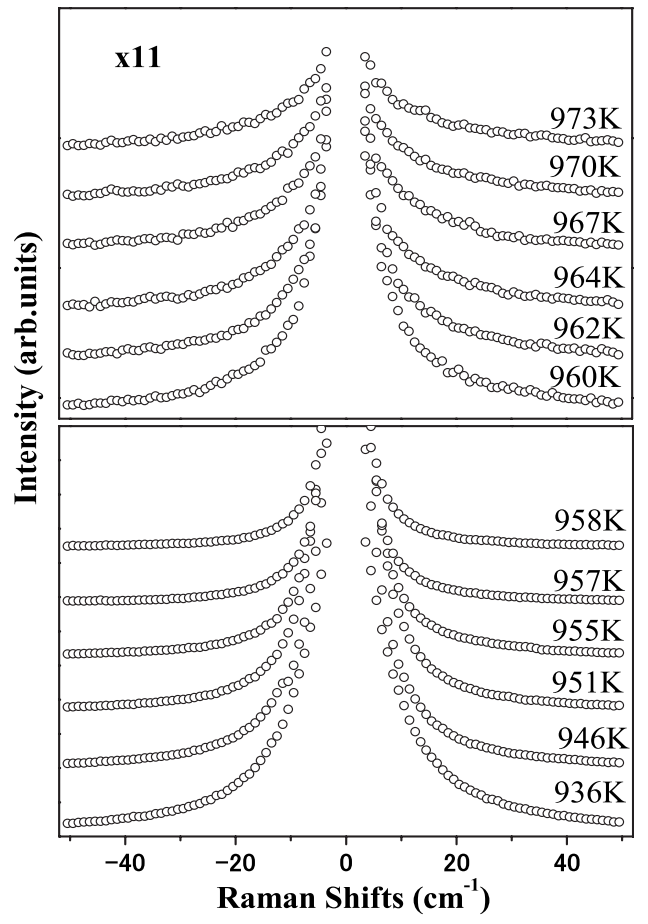

FIG. 5. Temperature dependence of the central peak at the $a(c, c) b$ scattering geometry measured by Raman spectroscopy. The spectra above $T_{C}$ are 11 times enlarged.

damping constant, the oscillator strength of the $i$ th mode, respectively. The population factor is $F(\nu, T)=[n(\nu)+1]$ or $F(\nu, T)=n(\nu)=1 /\left[\exp \left(h \nu / k_{B} T\right)-1\right]$ for the Stokes or antiStokes components of a spectrum, respectively.

When a relaxation process occurs, the spectral response $S(\nu, T)$ is usually approximated by the Debye relaxation

$$
S(\nu, T)=\chi^{\prime \prime}(\nu) F(\nu, T) \propto \frac{1}{1+\nu^{2} \tau^{2}} .
$$

$S(\nu, T)$ is approximated by a Lorentzian centered at zerofrequency shift. A half-width at half maximum is equal to the inverse of a relaxation time $\tau$. If there is a coupling between a damped harmonic oscillator and a Debye relaxation with an interaction strength $\delta^{2}$, the response function ${ }^{14}$ is given by

$$
\begin{aligned}
S(\nu, T)= & f_{0} \nu\left[\Gamma+\frac{\tau \delta^{2}}{1+4 \pi^{2} \nu^{2} \tau^{2}}\right] \\
& {\left[\nu_{0}^{2}-\frac{\delta^{2}}{1+4 \pi^{2} \nu^{2} \tau^{2}}-\nu^{2}\right]^{2}+\left[\Gamma+\frac{\tau \delta^{2}}{1+4 \pi^{2} \nu^{2} \tau^{2}}\right]^{2} \nu^{2} } \\
& \times F(\nu, T) .
\end{aligned}
$$

In the analysis of Raman spectra of the previous studies of CLT, different kinds of physical models were applied for CP. ${ }^{11,14,16}$ In the recent study of stoichiometric $\mathrm{LN},{ }^{30}$ the observed Raman spectra can be reproduced using either a sum of damped harmonic oscillators and a Debye-type relaxation mode or a damped harmonic oscillator with relaxing selfenergy. In the spectrum at RT, it is clear that $\mathrm{CP}, A_{1}\left(\mathrm{TO}_{1}\right)$, and the additional mode $a^{*}$ are clearly separated; therefore, it is not necessary to consider the coupling between the relax- 

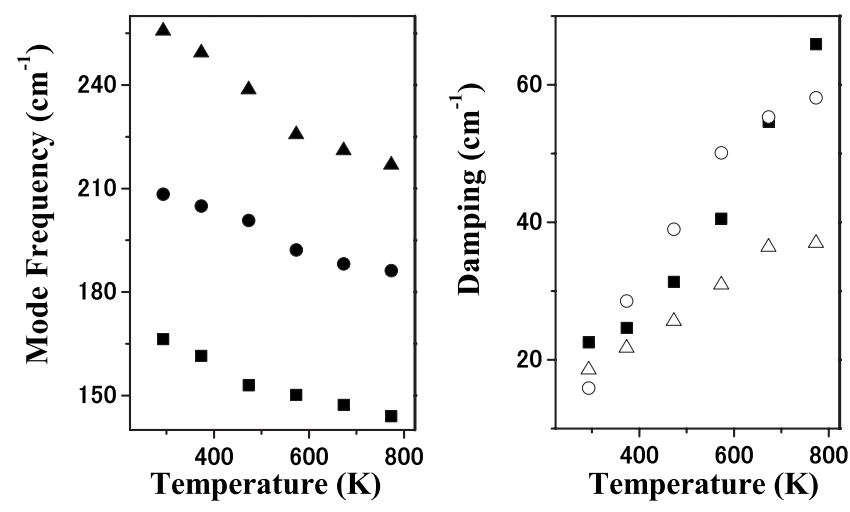

FIG. 6. Temperature dependence of the phonon frequency $\nu$ and damping $\gamma$ for the $a^{*}$ (solid squares) and the two lowest $A_{1}$ (TO) phonons, as deduced from the fit. Note that all the three modes are still not overdamped at a temperature of $773 \mathrm{~K}$.

ation mode and the normal phonon modes at least far below $T_{C}$. In our fitting procedure for the spectra from RT to $773 \mathrm{~K}$, we used Eqs. (3) and (4) and did not include any coupling. Figure 6 shows the frequency shift and the damping of the lowest three modes as a function of temperature. All these three modes show that the mode frequency decreases and the damping increases. It can be seen that the $A_{1}\left(\mathrm{TO}_{1}\right)$ mode is still underdamped at $773 \mathrm{~K}$. If we assume a displacive mechanism of a ferroelectric phase transition, the squared $A_{1}\left(\mathrm{TO}_{1}\right)$ mode frequency should follow a linear temperature dependence on $\left(T_{C}-T\right)$ as given by Eq. (1). However, the remarkable softening near $T_{C}$ is not observed. Therefore, it is certain that some kind of crossover to other mechanism may exist in the dynamics of the ferroelectric phase transition of LT.

On the other hand, between 773 and $873 \mathrm{~K}$, although the low-frequency modes can be observable, the strong $\mathrm{CP}$ does not allow any reliable determination of the $a^{*}$ and $A_{1}\left(\mathrm{TO}_{1}\right)$ frequency modes. Above $873 \mathrm{~K}$, the $a^{*}$ and $A_{1}\left(\mathrm{TO}_{1}\right)$ modes are hardly seen, and CP plays a dominant role. If one is interested only in $\mathrm{CP}$, a simplified but well working approach can be the fitting of $\mathrm{CP}$ by a single Debye relaxation, the same as the studies of CLT. ${ }^{11,15}$ Figures 7 and 8 show the temperature dependences of the relaxation time and the intensity of $\mathrm{CP}$ obtained by the single Debye fitting. The intensity shows gradual increase on heating up to $773 \mathrm{~K}$, while relaxation time decreases toward $900 \mathrm{~K}$, whereas above $900 \mathrm{~K}$ they drastically increase as the temperature approaches $T_{C}$, and the relaxation time diverges around $T_{C}$. The intensity of $\mathrm{CP}$ reaches its maximum around $T_{C}$ and then decreases for further heating. The inverse relaxation times near $T_{C}$ determined by the Raman and Brillouin scattering are shown in Fig. 9. It is found that below $T_{C}$, the inverse relaxation times are approximately proportional to $\left(T_{C}-T\right)$ as shown in Fig. 10. Such a phenomenon is well known as a critical slowing down of an order-disorder phase transition, and the relaxation time is described by Eq. (2). The fitted inverse relaxation time as a function of temperature in a ferroelectric phase gives the values of $\tau_{0}=20.80 \pm 0.04 \mathrm{fs}$ and $T_{C}=963.8 \pm 0.3 \mathrm{~K}$ for Raman scattering. For Brillouin scattering, the obtained parameters are $\tau_{0}=21.10 \pm 0.04 \mathrm{fs}$ and

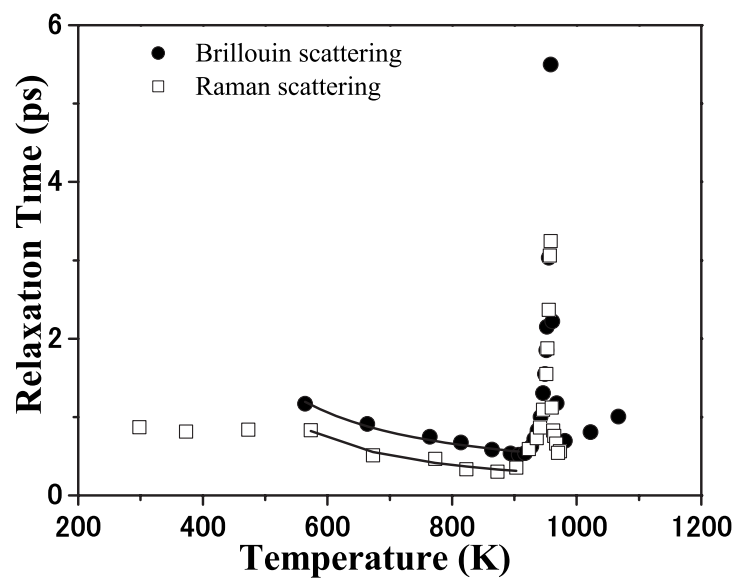

FIG. 7. Relaxation times of $\tau(T)$ in a nearly stoichiometric $\mathrm{LiTaO}_{3}$ crystal as a function of temperature determined by Brillouin and Raman scattering. Solid and open circles denote Brillouin and Raman data, respectively. It can be noted that the relaxation times obtained by both methods show critical slowing down near $T_{C}$. The two solid lines between 573 and $900 \mathrm{~K}$ denote the fitted Arrhenius behavior given by Eq. (6).

$T_{C}=961.8 \pm 0.3 \mathrm{~K}$. These results suggest that in the vicinity of $T_{C}$, the phase transition mechanism of SLT is well described by the order-disorder mechanism.

\section{E. Origin of central peak}

In the studies of structural phase transitions, several different origins were discussed on CPs observed by Raman and Brillouin scattering. The most important distinction of the origin of $\mathrm{CP}$ is whether it is static or dynamic. The static origin gives rise to an elastic unresolved and temperatureindependent $\mathrm{CP}$, while the dynamic origin results in a relatively broad and temperature-dependent CP. The detailed distinctions among various dynamic mechanisms are related to its temperature, symmetry, and wave vector dependences. ${ }^{31-39}$ Concerning CP of CLT, several mechanisms were discussed. ${ }^{11,12,14,16}$ Penna et al. ${ }^{11,12}$ showed that

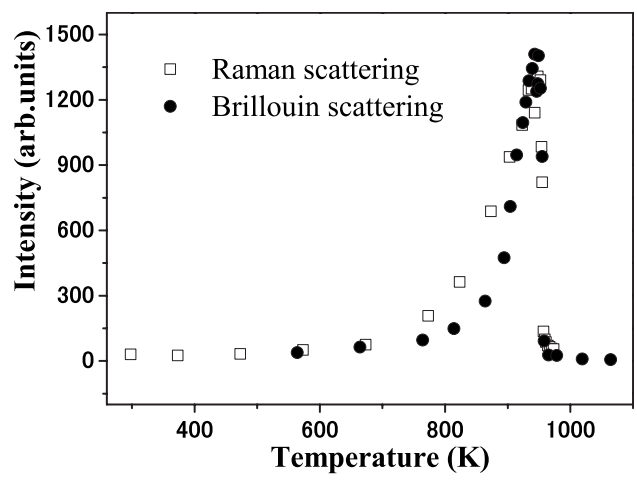

FIG. 8. Integrated intensity of the central peaks as a function of temperature obtained by Brillouin and Raman scattering method. Solid circles are the Brillouin data; open squares are the Raman data. It can be noted that the integrated intensity obtained by both scattering shows the peak at $T_{C}$. 


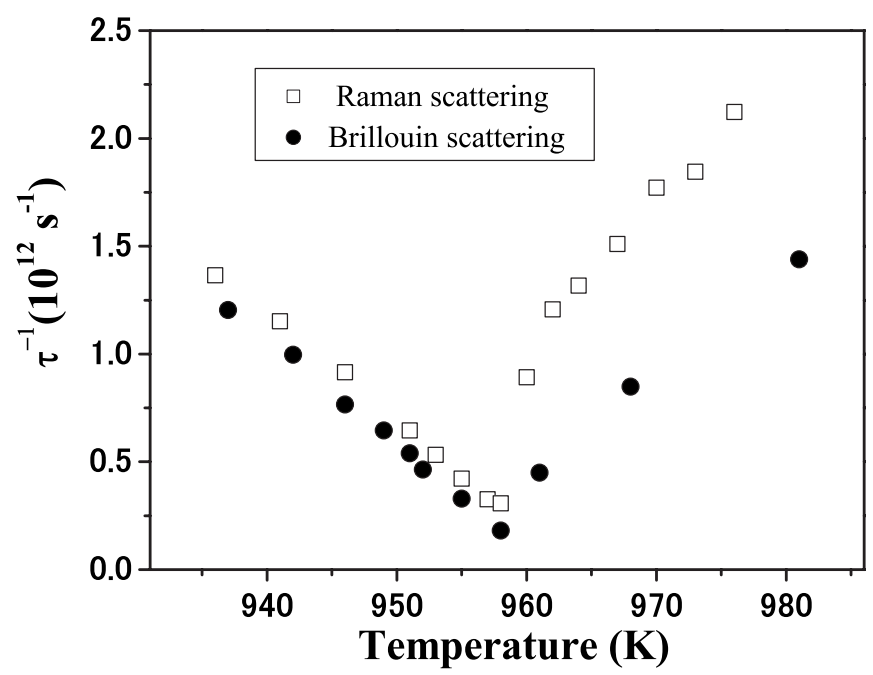

FIG. 9. Temperature dependence of the inverse relaxation time in the vicinity of $T_{C}$ obtained by Brillouin and Raman scattering. Solid circles are the Brillouin data; open squares are the Raman data.

temperature-dependent dynamical domain fluctuations are responsible for CP. However, as indicated in Refs. 40 and 41 ultrasonic measurements clarified the distinction between single domain and multidomain states in CLT just below $T_{C}$, and furthermore, the dielectric dispersion was observed in a paraelectric phase of CLT. Consequently, the domain fluctuation model is inconsistent with the elastic and dielectric properties. Zhang and $\mathrm{Scott}^{14}$ fitted their Raman spectra by Eq. (5) and concluded that both soft-mode linewidths and coupling constant are controlled by the same microscopic extrinsic process related to the oxygen vacancies. They further discussed that these defects were characterized by relaxation times that continue to become longer above $T_{C}$. There-

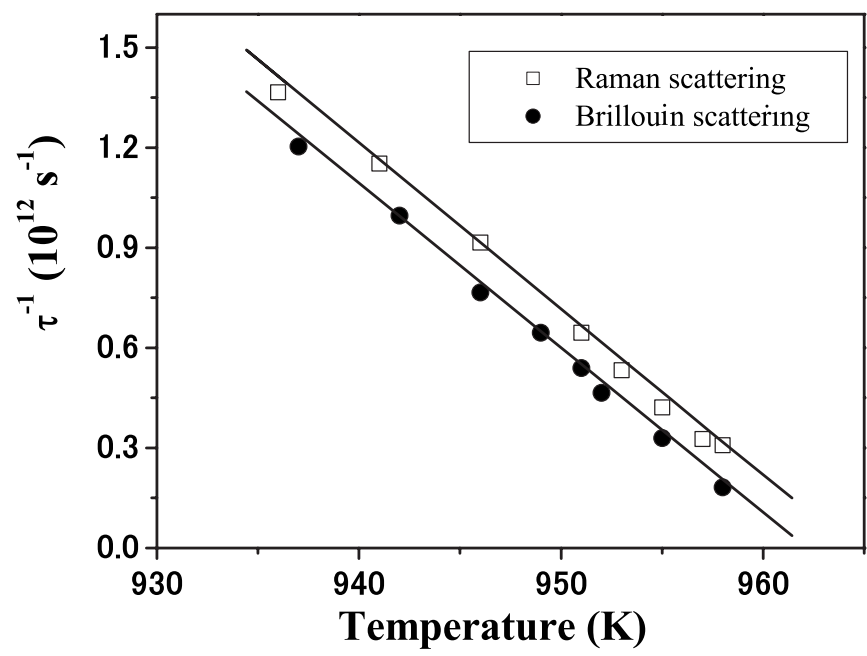

FIG. 10. Inverse relaxation time of a stoichiometric $\mathrm{LiTaO}_{3}$ crystal as a function of temperature. Solid circles are the Brillouin data; open squares are the Raman data. Solid curve is a fit to Eq. (2) with $T_{C}=961.8 \mathrm{~K}$ and $\tau_{0}=21.1 \mathrm{fs}$ for Brillouin scattering and $T_{C}$ $=963.8 \mathrm{~K}$ and $\tau_{0}=20.8 \mathrm{fs}$ for Raman scattering. fore, these vacancies are independent on the transition mechanism.

In contrast, present results of SLT are not consistent with the above arguments for the following reasons. First, the relaxation time diverges at $T_{C}$ and shows a critical slowing down both above and below $T_{C}$. Second, our measurement was carried out using the nearly stoichiometric LT, which has very small defect density in comparison with those of CLT; nevertheless, a very intense CP is observed. Halperin and Varma ${ }^{39}$ discussed the influence of defects on the phase transition mechanism and showed that the interaction strength of coupling $\delta^{2}$ in the coupled mode analysis is proportional to the defect concentration. Zhang and Scott ${ }^{14}$ pointed out that the parameter $\delta^{2}$ obtained from the fitting is ambiguous in comparison with the effective coupling parameter $\delta^{2} \tau$ in their study. We also fitted our high temperature spectra by Eq. (5). The obtained parameter $\delta^{2} \tau$ for a spectrum at $823 \mathrm{~K}$ is $180 \pm 5 \mathrm{~cm}^{-1}$, which is very close to the value at the corresponding temperature in Ref. 14. This means that the strong CPs of SLT observed in the present study have different dynamic origin from those of CLT. The temperature dependences of CPs observed by Brillouin and Raman scattering are shown in Figs. 7 and 8, respectively. The temperature dependence of the relaxation time obeys Eq. (2) of orderdisorder nature as shown in Figs. 9 and 10. Consequently, it is concluded that the origin of CP of SLT near $T_{C}$ is completely different from that of CP of CLT.

Although we did not observe any $\mathrm{CP}$ in $E(x, y)$ spectra at room temperature, above $723 \mathrm{~K}$, we observed a very weak $\mathrm{CP}$ in comparison with that observed in $A_{1}(z)$ spectra. Its intensity increases markedly toward $T_{C}$ and shows similar temperature behavior as those observed at the $a(c, c) b$ scattering geometry. The relaxation time also shows critical slowing down near $T_{C}$ as shown in Fig. 11. The large fluctuations of the $\mathrm{Li}$ ion motion in the very vicinity of $T_{C}$ may cause the dynamical structural disorder, and they may break local crystal symmetry dynamically and enhance the leakage of $\mathrm{CP}$ into the depolarized component.

The integrated intensity of diffuse scattering and CP obtained by the neutron, Brillouin, and Raman scattering is shown in Fig. 12. We note the sharp decrease of the CP intensity just above $T_{C}$ in light scattering and the gentle decrease in neutron scattering. The $\mathrm{CP}$ intensity of uniaxial relaxor single crystals of $\mathrm{Sr}_{0.61} \mathrm{Ba}_{0.39} \mathrm{Nb}_{2} \mathrm{O}_{6}$ gives a nearly symmetrical change around $T_{C}{ }^{42}$ The selection rule does not hold for the relaxor ferroelectric, while LT is a normal ferroelectric material. Thus, the asymmetrical temperature change of CP intensity of SLT in light scattering is attributed to the fact that the polarization fluctuation along the ferroelectric $c$ axis is Raman inactive above $T_{C}$. Consequently, these behaviors of $\mathrm{CP}$ indicate that the polarization fluctuations along a ferroelectric $c$ axis plays a dominant role in CP.

\section{F. Dynamics of Li ions}

Abrahams et al. found in a PE phase that a Li ion randomly distributes over either position of equivalent sites that locate both sides of an oxygen plane, while a Ta ion locates at an inversion center. ${ }^{18}$ If a $\mathrm{Li}$ ion is flipping between these 

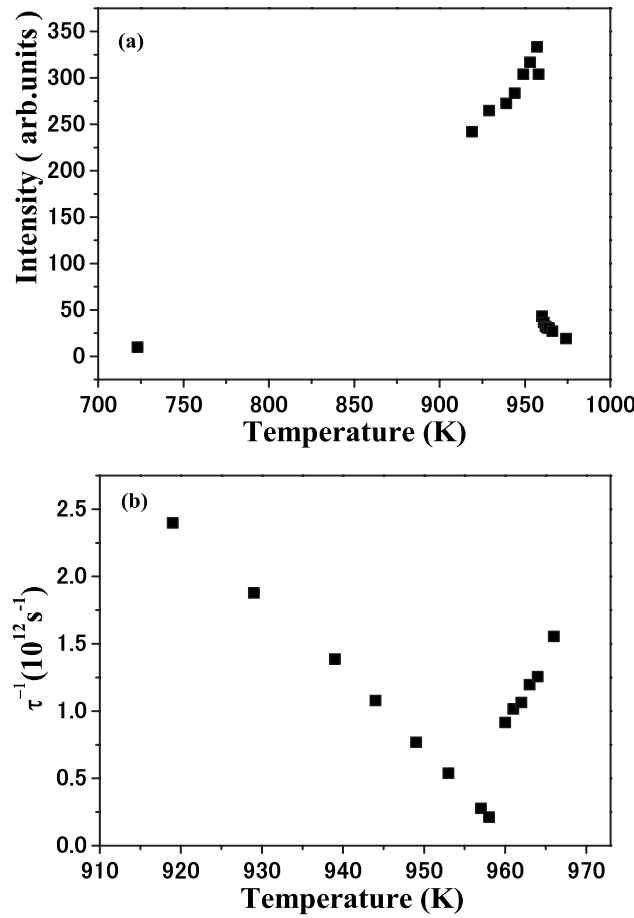

FIG. 11. The fitting parameters of the central peaks of VH Raman scattering observed at the $a(c, a) b$ scattering geometry. (a) The temperature dependence of the integrated intensity. (b) The temperature dependence of the inverse relaxation time near $T_{C}$. It can be seen that relaxation time shows critical slowing down.

two equivalent sites, $\mathrm{CP}$ resulting from such motion can appear in a light scattering spectrum. Such CP was reported on Raman scattering studies of several classical pure and mixed perovskite compounds such as $\mathrm{KNbO}_{3}, \mathrm{BaTiO}_{3}$, and $\mathrm{KTa}_{1-x} \mathrm{Nb}_{x} \mathrm{O}_{3},{ }^{37,38}$ and also on Brillouin scattering of the new ferroelectric $\mathrm{BaTi}_{2} \mathrm{O}_{5}$, which shows a typical critical slowing down of an order-disorder phase transition. ${ }^{43,44}$ Such a CP has a Lorentzian-like shape centered at the zerofrequency shift that is a typical feature of a Debye relaxation.

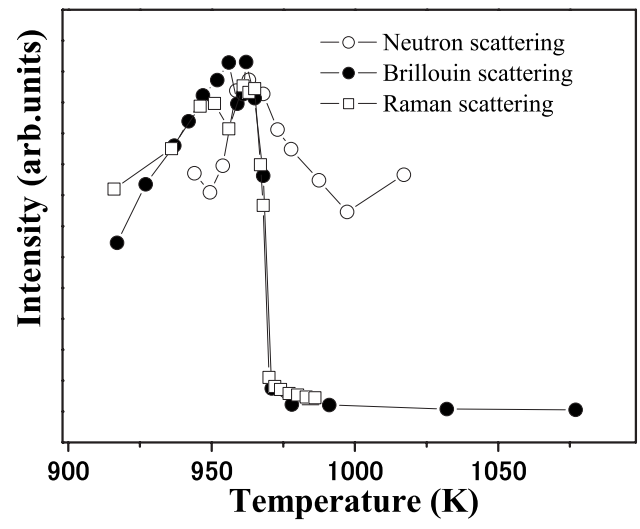

FIG. 12. The temperature dependences of the integrated intensity obtained by neutron, Brillouin, and Raman scattering. Open circles are the neutron data taken in the vicinity of the $(0,0,6)$ Bragg peak. Solid circles are the Brillouin data. Open squares are the Raman data. The CP intensity of light scattering drops sharply just above $T_{C}$, while that of neutron scattering decreases gradually.
These relaxation times are the same order as we observed in SLT.

Birnie $^{45}$ explained the temperature dependence of polarization of CLT reported in Ref. 46 by the order-disorder model. As temperature increases from RT, Li ions become disorder between their normal sites and the normally vacant octahedral sites. The difference in the energy between two kinds of sites determines the relative site occupancy of each site. Any different probability of occupancy between two sites causes some net spontaneous polarization in the crystal lattice, which, in turn, enhances more ordering. Such dynamics can be described by the local energy barrier height $U$ between two sites. The relaxation time $\tau$ obeys the Arrhenius law

$$
\tau=\tau_{0} \exp \left(\frac{U}{k_{B} T}\right),
$$

where $\tau_{0}$ is the attempt relaxation time. In a Brillouin spectrum at $a(c, c) b$ geometry, the intensity of $\mathrm{CP}$ is very weak at $\mathrm{RT}$, and the relaxation time is of the same order as those observed below $900 \mathrm{~K}$. However, the weak intensity does not allow us to get reliable fitting parameters below $564 \mathrm{~K}$. In a Raman spectrum, we can determine the parameters with a small fitting error, while the weak intensity of $\mathrm{CP}$ causes the difficulty in discussing the temperature dependence of the relaxation time also below $573 \mathrm{~K}$. We fitted the relaxation time by Eq. (6) in the range of 573-903 K for Raman scattering and 564-907 K for Brillouin scattering spectra. The solid lines in Fig. 7 show the Arrhenius law with the parameters $\tau_{0}=161 \pm 16 \mathrm{fs}, U / k_{B}=1132 \pm 70 \mathrm{~K}$ for Brillouin scattering and with $\tau_{0}=58.6 \pm 13 \mathrm{fs}, U / k_{B}=1512 \pm 152 \mathrm{~K}$ for Raman scattering. The difference exists between the results of Brillouin data and the Raman data. In Raman scattering, the deviation may come from the contribution of the lowfrequency optical phonon. These results indicate that relative site occupancy of the Li ions occurs between the two sites in the temperature range of 573-903 K, and the energy barrier height is obtained as $97.5 \pm 6 \mathrm{meV}$ by the fitting of Brillouin spectra using Eq. (6). Therefore, $\mathrm{CP}$ far below $T_{C}$ does not reflect the order-disorder nature of a phase transition. In contrast, at higher temperatures above $903 \mathrm{~K}$, different dynamics must be taken into account. In fact, above $903 \mathrm{~K}$, the relaxation time starts to increase, and near the vicinity of $T_{C}$, it shows critical slowing down of an order-disorder transition as discussed in Sec. III D.

\section{CONCLUSION}

The ferroelectric phase transition of nearly stoichiometric $\mathrm{LiTaO}_{3}$ crystals has been studied by Raman, Brillouin, and neutron scattering to investigate CPs in much extended spectral and temperature ranges. The temperature dependence of order parameter determined by the $(1,1,12)$ Bragg peak intensity shows the high value of the Curie temperature $T_{C}$, which is the typical feature of the nearly stoichiometric composition.

CP observed by Raman scattering has the strong polarization dependence and is observed only in $A_{1}(z)$ spectra except near $T_{C}$. These spectra have been fitted by a damped har- 
monic oscillator and a Debye relaxation mode without any coupling. The fitting shows that the $A_{1}(z)$ phonon frequency decreases and its damping increases with the increase of temperature from the room temperature. However, in the vicinity of $T_{C}$, the complete softening of the lowest mode is never observed. While in the near vicinity of $T_{C}, \mathrm{CP}$ exhibits a critical slowing down of an order-disorder phase transition. It is found that the crossover from the displacive to the orderdisorder nature occurs near $T_{C}$.

\section{ACKNOWLEDGMENTS}

This research was partially supported by the Grant-in-Aid for Scientific Research (A), 2005, 16204032, and in part by the 21st Century COE program under MEXT, Japan. Neutron measurements were performed at the Swiss spallation neutron source SINQ, Paul Scherrer Institut, Villigen, Switzerland.
*On leave from Ioffe Physical Technical Institute, 26 Politekhnicheskaya, 194021, St. Petersburg, Russia.

†kojima@bk.tsukuba.ac.jp

${ }^{1}$ W. Cochran, Phys. Rev. Lett. 3, 412 (1959).

${ }^{2}$ M. E. Lines and A. M. Glass, Principles and Application of Ferroelectrics and Related Materials (Clarendon, Oxford, 1977).

${ }^{3}$ T. Riste, E. J. Samuelsoen, K. Olsen, and J. Feder, Solid State Commun. 9, 1455 (1971).

${ }^{4}$ R. A. Cowley, G. J. Coombs, R. S. Katiyar, J. F. Ryan, and J. F. Scott, J. Phys. C 4, L203 (1971).

${ }^{5}$ B. Zalar, V. V. Laguta, and R. Blinc, Phys. Rev. Lett. 90, 037601 (2003).

${ }^{6} \mathrm{Y} . \mathrm{Xu}$, Ferroelectric Materials and Their Applications (NorthHolland, Amsterdam, 1991).

${ }^{7}$ S. C. Abrahams, W. C. Hamilton, and A. Sequeira, J. Phys. Chem. Solids 28, 1963 (1967).

${ }^{8}$ S. C. Abrahams and J. L. Bernstein, J. Phys. Chem. Solids 28, 1685 (1967).

${ }^{9}$ W. D. Johnston, Jr. and I. P. Kaminov, Phys. Rev. 168, 1045 (1968).

${ }^{10}$ M. E. Lines, Solid State Commun. 10, 793 (1972).

${ }^{11}$ A. F. Penna, A. Chaves, and S. P. S. Porto, Solid State Commun. 19, 491 (1976).

${ }^{12}$ A. F. Penna, S. P. S. Porto, and E. Wiener-Avnear, Solid State Commun. 23, 377 (1977).

${ }^{13}$ J. L. Servoin and F. Gervais, Solid State Commun. 31, 387 (1979).

${ }^{14}$ M. S. Zhang and J. F. Scott, Phys. Rev. B 34, 1880 (1986).

${ }^{15}$ C. Raptis, Phys. Rev. B 38, 10007 (1988).

${ }^{16}$ Y. Tezuka, S. Shin and M. Ishigame, Phys. Rev. B 49, 9312 (1994).

${ }^{17}$ G. Cheng, B. Hennion, P. Launois, X. L. Meng, B. Ch. Xu, and Y. M. Jiang, J. Phys.: Condens. Matter 5, 2707 (1993).

${ }^{18}$ S. C. Abrahams, E. Buehler, W. C. Hamilton, and S. J. Laplaca, J. Phys. Chem. Solids 34, 521 (1973).

${ }^{19}$ G. P. Wiederrecht, T. P. Dougherty, L. Dhar, K. A. Nelson, D. E. Leaird, and A. M. Weiner, Phys. Rev. B 51, 916 (1995).

${ }^{20}$ T. F. Crimins, N. S. Stoyanov, and K. A. Nelson, J. Chem. Phys. 117, 2882 (2002).

${ }^{21}$ S. Kojima, H. Kitahara, S. Nishizawa, and M. W. Takeda, Jpn. J. Appl. Phys., Part 1 42, 6238 (2003).

${ }^{22}$ J. F. Araujo, J. M. Filho, F. E. A. Melo, F. V. Letelier, and A. S. Chaves, Phys. Rev. B 57, 783 (1998).

${ }^{23}$ A. M. Pugachev, H. Anwar, and S. Kojima, Phys. Status Solidi C
1, 3122 (2004).

${ }^{24}$ P. Böni and P. Keller, Proceedings of the Fourth Summer School on Neutron Scattering, Zuoz, Switzerland, 18-24 August 1996 (unpublished), pp. 35-42.

${ }^{25}$ W. E. Fischer, Physica B 234-236, 1202 (1997).

${ }^{26}$ X. Yang, G. Lan, B. Li, and H. Huang, Phys. Status Solidi B 141, 287 (1987)

${ }^{27}$ S. Kojima, Jpn. J. Appl. Phys., Part 1 32, 4373 (1993).

${ }^{28}$ A. Ridah, P. Bourson, M. D. Fontana, and G. Malovichko, J. Phys.: Condens. Matter 9, 9687 (1997).

${ }^{29}$ A. Ridah, M. D. Fontana, and P. Bourson, Phys. Rev. B 56, 5967 (1997).

${ }^{30}$ N. V. Surovtsev, A. M. Pugachev, V. K. Malinovsky, A. P. Shebanin, and S. Kojima, Phys. Rev. B 72, 104303 (2005).

${ }^{31}$ P. A. Fleury and K. B. Lyons, Solid State Commun. 32, 103 (1979).

${ }^{32}$ P. A. Fleury and K. B. Lyons, in Light Scattering Near Phase Transitions, edited by H. Z. Cummins and A. P. Levanyuk (North-Holland, Amsterdam, 1983).

${ }^{33}$ S. Furusawa, T. Suemoto, and M. Ishigame, Phys. Rev. B 38, 12600 (1988).

${ }^{34}$ B. Mohammadou, G. E. Kugel, F. Brehat, B. Wyncke, G. Mornier, and P. Simons, J. Phys.: Condens. Matter 3, 9489 (1991).

${ }^{35}$ T. Shima, M. Kasahara, P. Kaung, and T. Yagi, J. Phys. Soc. Jpn. 65, 1102 (1996).

${ }^{36}$ Y. Tsujimi, T. Matsui, H. Furuta, and T. Yagi, Phys. Rev. B 59, 28 (1999).

${ }^{37}$ J. P. Sokoloff, L. L. Chase, and D. Rytz, Phys. Rev. B 38, 597 (1988).

${ }^{38}$ J. P. Sokoloff, L. L. Chase, and L. A. Boatner, Phys. Rev. B 41, 2398 (1990).

${ }^{39}$ B. I. Halperin and C. M. Varma, Phys. Rev. B 14, 4030 (1976).

${ }^{40}$ I. Tomeno and S. Matsumura, Phys. Rev. B 38, 606 (1988).

${ }^{41}$ I. Tomeno, J. Phys. Soc. Jpn. 51, 2891 (1982).

${ }^{42}$ F. M. Jiang, J.-H. Ko, and S. Kojima, Phys. Rev. B 66, 184301 (2002).

${ }^{43}$ A. Hushur, H. Shigematsu, Y. Akishige, and S. Kojima, Jpn. J. Appl. Phys., Part 1 43, 6825 (2004).

${ }^{44}$ A. Hushur, H. Shigematsu, Y. Akishige, and S. Kojima, Appl. Phys. Lett. 86, 112903 (2005).

${ }^{45}$ Dunbar P. Birnie III, J. Appl. Phys. 69, 2485 (1991).

${ }^{46}$ A. M. Glass, Phys. Rev. 172, 564 (1968). 